\title{
Rescan Time Delays in Ischemic Stroke Imaging: A Retrospective Observation and Analysis of Causes and Clinical Impact
}

(D).M. Katz, (D).J. Wang, (D)A.T. Boltyenkov, (D) G. Martinez, (D). O’Hara, (D). Feizullayeva, (D) M. Gribko, (D)A. Pandya, and

(iD) P.C. Sanelli

\begin{abstract}
BACKGROUND AND PURPOSE: Delays to reperfusion negatively impact outcomes of patients with ischemic stroke, yet current guidelines recommend selective sequential imaging for thrombectomy candidates. We aimed to quantify and analyze time delays associated with rescanning in sequential acute stroke imaging.
\end{abstract}

MATERIALS AND METHODS: This was a retrospective cohort study of consecutive patients with acute ischemic stroke who underwent imaging for treatment decision-making from January 1, 2017, to June 30, 2020. Rescan time delay was defined as $\geq 10$-minute difference between initial NCCT and CTA \pm CTP. Mean rescan time delays in comprehensive and primary stroke centers were compared. Bivariate and multivariable regression analyses assessed clinical and imaging factors associated with rescanning time delays and early outcomes.

RESULTS: A total of 588 patients with acute ischemic were included in statistical analyses. Rescanning occurred in 27.9\% (164/588 patients), with a mean time delay of 53.7 (SD, 43.4) minutes. For patients presenting at primary compared with comprehensive stroke centers, rescan time delays were more common (59.6\% versus $11.8 \%, P<.001)$, with longer delays $(65.4$ [SD, 45.4 ] minutes versus 23.6 [SD, 14.0] minutes, $P<.001)$. Independent predictors of rescan time delays included primary stroke center presentation, intravenous thrombolysis administration, black race, admission NIHSS $\geq 10$, baseline independent ambulation, and onset-tocomprehensive stroke center arrival in $\geq 6$ hours. Protocols for early simultaneous comprehensive CT (NCCT + CTA + CTP) were associated with lower odds of time delays $(\mathrm{OR}=0.34 ; 95 \% \mathrm{Cl}, 0.21-0.55)$. Rescanning was associated with lower odds of home discharge (OR $=0.53 ; 95 \% \mathrm{Cl}, 0.30-0.95)$.

CONCLUSIONS: A sequential approach to CT-based imaging may be significantly associated with prolonged acute stroke evaluations. Adoption of early simultaneous comprehensive CT could minimize treatment delays and improve outcomes.

ABBREVIATIONS: CTAP = CTA perfusion; CSC-G = comprehensive stroke center imaging subgroup; PSC-G = primary stroke center imaging subgroup

$\mathbf{T}$ reating select patients with acute ischemic stroke with endovascular thrombectomy up to 24 hours from last known well increases the proportion of patients with large-vessel occlusion who have an independent functional outcome. ${ }^{1-7}$ The robust benefits of endovascular stroke treatment have been shown to be timedependent in numerous analyses of randomized clinical trials and a large registry, in which the benefits decrease as time to reperfusion increases in a nonlinear fashion. ${ }^{8-11}$ Stroke systems of care initiatives

Received March 5, 2021; accepted after revision May 3.

From the Departments of Neurology (J.M.K., M.G.) and Radiology (J.M.K., A.T.B., G.M., P.C.S.), Donald and Barbara Zucker School of Medicine at Hofstra/Northwell, Hempstead, New York; Feinstein Institutes for Medical Research (J.J.W., A.T.B., G.M. J.O., C.F., P.C.S.), Manhasset, New York; Siemens Medical Solutions (A.T.B., G.M.), Malvern, Pennsylvania; and Department of Health Policy and Management (A.P.), Harvard T.H. Chan School of Public Health, Boston, Massachusetts.

This work was supported by the Feinstein Institutes for Medical Research, Northwell Health, the National Institute of Neurological Disorders and Stroke of the National Institutes of Health under award number R56NS114275, and Siemens.

The content is solely the responsibility of the authors and does not necessarily represent the official views of the National Institutes of Health. focus on improving the efficiency of acute stroke management to maximize favorable clinical outcomes. For instance, the American Heart Association Target: Stroke Phase III initiative (https://www. heart.org/en/professional/quality-improvement/target-stroke/ introducing-target-stroke-phase-iii) challenges comprehensive stroke centers to achieve door-to-device times of $<60$ minutes for transfer patients and $<90$ minutes for direct emergency department arrivals. $^{12}$

The most recent American Heart Association guidelines for the management of patients with acute ischemic stroke recommend NCCT of the head as the initial imaging technique for

Please address correspondence to Jeffrey M. Katz, MD, Department of Neurology, Northwell Health, 611 Northern Blvd, Great Neck, NY 11021; e-mail: jkatz2@northwell.edu

- Indicates open access to non-subscribers at www.ajnr.org

Indicates article with online supplemental data.

http://dx.doi.org/10.3174/ajnr.A7227 
triage of patients with acute stroke. ${ }^{13}$ Comprehensive CT imaging with head and neck CTA and perfusion CTA (CTAP) is recommended specifically for patients who may be endovascular candidates, with CTP only for patients in the $>6$ - to 24-hour time window. Consequently, imaging practices have evolved to perform initial NCCT first to rapidly determine eligibility for intravenous thrombolysis; then, patients are returned to the scanner for CTA \pm CTP, particularly when thrombectomy is considered. $^{13-15}$ The main concern is that these inefficient imaging practices have potentially led to unnecessary time delays to endovascular treatment, translating to worse clinical outcomes. ${ }^{16-19}$ While there have been several studies that report the mean time it takes to rescan patients with acute stroke with urgent MR imaging following an initial NCCT or CTAP in an acute trial setting, ${ }^{20-23}$ no study to date has quantified the time delay associated with rescanning for advanced CT-based imaging following an initial NCCT in real-world practice.

In this study, we aimed to quantify the time it takes to rescan patients with acute ischemic stroke with CTA \pm CTP following NCCT at comprehensive and primary stroke centers within the same stroke network. We then assessed the clinical and imaging factors associated with rescan time delays and analyzed whether these delays impacted early clinical outcomes. Our hypothesis was that patients with rescan time delays will have worse clinical outcomes.

\section{MATERIALS AND METHODS}

This was a retrospective cohort study of consecutive patients with acute ischemic stroke admitted to the North Shore University Hospital comprehensive stroke center between January 1, 2017, and June 30, 2020. Institutional review board approval was obtained with a waiver of consent because of the retrospective nature of this study. Demographic, clinical, treatment, and outcome data were imported from the Get-With-TheGuidelines-Stroke data base (https:/www.clinicaltrials.gov/ct2/ show/NCT02693223) into a separate Stroke Health Outcomes database. The Stroke Health Outcomes database incorporates additional patient-level details regarding imaging performed during the index stroke admission, identified by unique identifiers in the health system's PACS. Imaging examinations were collected chronologically from 48 hours prearrival from another institution (ie, primary stroke center) until the discharge date, with each imaging examination assigned as a separate entry in the patient's record in the Stroke Health Outcomes database.

Because rescan time delays predominantly affect patients with acute stroke who undergo treatment with intravenous thrombolysis and/or endovascular thrombectomy, the study inclusion aimed to select patients with the following criteria: patients with suspected acute stroke who were likely considered potential treatment candidates and underwent early comprehensive CT imaging with CTA \pm CTP, with the likely intent of evaluating for acute stroke intervention and within treatment decision-making timeframes sufficient to meet the Target: Stroke Phase III initiative's 90-minute door-to-device goal. ${ }^{12}$ Our imaging timeframe criteria further aimed to exclude patients being imaged only for management purposes in secondary stroke prevention. Therefore, taking a more conservative approach, we chose different imaging timeframes, depending on the mode of arrival to the comprehensive stroke center, to increase the accuracy of patient inclusion in the study: 1) Direct arrivals had to have the first NCCT and CTA or CTAP performed within 1 hour of hospital arrival to differentiate patients who were potential treatment candidates from those being imaged for secondary stroke prevention management decisions. 2) Transfer patients from a primary stroke center had to be transferred for thrombectomy evaluation and to have undergone CTA at the referring site. To confirm this process, we required that transfer patients meet additional criteria, including the following: 1) NCCT and CTA were performed at the referring site to assess thrombectomy eligibility within the 6 hours before comprehensive stroke center door time, and 2) CTP (only performed for thrombectomy evaluations at our institution) was the first imaging completed at the comprehensive stroke center within 1 hour of arrival. Direct arrivals defined the comprehensive stroke center imaging subgroup (CSC-G), and transfer patients constituted the primary stroke center imaging subgroup (PSC-G). Exclusion criteria were patients with acute stroke younger than 18 years of age and incomplete imaging data. A flow diagram illustrating the final selection of patients for inclusion in the statistical analysis is shown in Fig 1.

Data collection included the type of imaging examination (NCCT, CTA, CTP) and the date and earliest imaging timestamp in the PACS. The recorded timestamps reference the start of the imaging performance. However, when multiple imaging examinations were completed in a single scanning session and assigned the same accession number, the earliest timestamp was used for all examinations within the imaging session. Only the pretransfer imaging timestamps were used for the PSC-G to exclude the transfer time to the comprehensive stroke center in the rescan time. Imaging during off-hours was defined as overnight (5:00 PM to 8:00 AM) and weekend days. Other data included demographic characteristics (age, sex, race [black, white, Asian, unknown/other], health insurance [private, Medicare/Medicaid, uninsured/self-pay], median household income $[<\$ 80,000$, $\$ 80,000-\$ 120,000$, and $>\$ 120,000$, based on ZIP code and census data]); ${ }^{24}$ clinical factors (admission NIHSS score, arrival mode [ambulance, private transportation] and time [year, day, time], onset-to-arrival time [defined as last known well to comprehensive stroke center door time], treatment [intravenous thrombolysis, thrombectomy, none], length of hospital stay [0-7 days and $\geq 8$ days], and the time interval between NCCT and CTA \pm CTP imaging); and early outcomes (discharge disposition [home, rehabilitation facility, died/hospice], and discharge mRS). On the basis of the discharge mRS scores, good clinical outcome was defined as functional independence (mRS 0-2) at discharge. Age was dichotomized as younger than 80 years versus 80 years of age or older because of the negative impact of older age ( 80 years of age or older) on stroke outcomes. ${ }^{25,26}$ Data collection on race, based on self-reported identification of racial categories, and analyses of racial disparities in healthcare delivery, was included in this study according to National Institutes of Health policy ${ }^{27}$ and the American Heart Association position statement recommendations. ${ }^{28}$

During the study period, CTP was rarely performed at the primary stroke centers in our healthcare system. At the comprehensive stroke center, CTP was first adopted for evaluating selected patients for thrombectomy candidacy in late 2017 on the basis of 


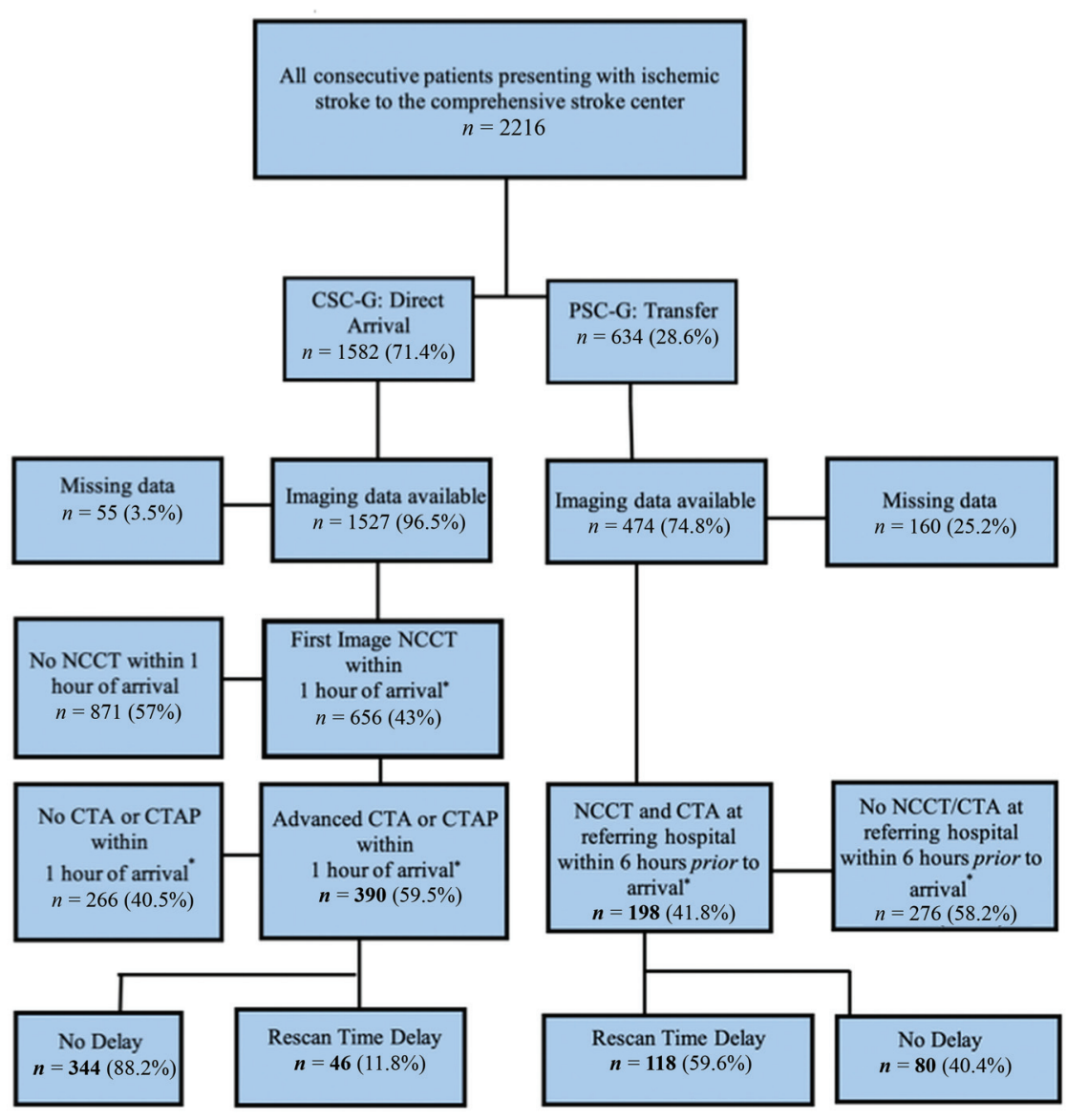

FIG 1. Flowchart of patient selection. "Arrival asterisk" refers to door time at the comprehensive stroke center.

the results of the first extended time window thrombectomy trial. ${ }^{6}$ In 2019, acute stroke imaging with NCCT and CTAP during the same session became protocolized at the comprehensive stroke center as routine imaging for virtually all patients with stroke presenting within 24 hours of last known well. Similarly, in 2019, owing to adoption of local early comprehensive CT imaging protocols at many of the primary stroke centers, CTA performance in tandem with the NCCT at these referring sites became more routine. To reflect these protocol changes in our analyses, admission year was dichotomized into pre- (2017 and 2018) and postprotocol (2019 and 2020) implementation time periods.

\section{Statistical Analysis}

For comparative analyses, we classified patients with and without rescan time delays, defined as a difference of $\geq 10$ minutes between the initial NCCT and CTA \pm CTP. Ten minutes was chosen as a cutoff threshold because of the following: 1) Patients with simultaneous comprehensive CT imaging (CTAP) have a single accession number for all studies and will have a zerominute time difference between the initial NCCT and CTA \pm CTP examinations; 2) we wanted to exclude single-session imaging with different accession numbers from the time-delay group, a situation that may occur when multiple orders are placed separately; and 3) based on our experience, a 10-minute time interval allows the additional time that may be required in some patients to set up the next scanning protocol, intravenous access issues, and administration of intravenous thrombolytics on the table when appropriate. Our selection of 10 minutes as the threshold defining time delay is further supported by previous literature reporting median time intervals of 6 minutes $^{29}$ (CT + CTA) and 12 minutes $^{30}$ (CTAP) for acquisition of single-session comprehensive CT imaging in patients with acute ischemic stroke, with the latter study including time for CTP processing and analysis. For the CSC-G, the rescan time delay was calculated as the time difference between the initial NCCT and either CTA or CTAP. When NCCT and CTA were performed in the same session and CTP was in a separate session (within 60 minutes from NCCT), then the difference between NCCT-CTA and CTP was used. For the PSC-G, rescan time delay was defined as the difference between NCCT and CTA at the primary stroke center before transfer.

For the primary analyses, we combined the CSC-G and PSC-G data to stratify patients with and without rescan time delays ( $\geq 10$ minutes). The demographic characteristics, clinical factors, and early outcomes in patients with and without rescan time delays were compared using bivariate analyses. Independent-samples $t$ tests and rank sum tests analyzed continuous variables, and $\chi^{2}$ tests analyzed categoric variables. Multivariable logistic regression was performed to separately analyze the association between demographic/clinical parameters and rescan time delays, and rescan time delays plus demographic/clinical parameters and early outcomes. All statistically significant variables from the bivariate analyses and clinically important factors, such as age, sex, median household income, and onset-to-arrival time of $>6$ hours, were included in the regression models. When $>2$ subcategories of a categoric variable were available to move forward into the multivariable logistic regression analysis, we selected either the variable with the largest difference between groups (from the bivariate analysis), or when numeric differences were small, we selected the subcategory hypothesized to be most clinically relevant, with the other subcategories combined into the reference category. The CochranMantel-Haenszel shift test was used to perform mRS and discharge disposition shift analyses for different time-delay intervals.

Secondary analyses were then performed to assess selection bias that may have influenced our primary results. We applied a uniform 1- or 6-hour door-to-imaging window limit to select 


\begin{tabular}{|c|c|c|c|c|}
\hline \multirow[b]{2}{*}{ Parameter } & \multicolumn{2}{|c|}{ Rescan Time Delay } & \multicolumn{2}{|c|}{ Home Discharge } \\
\hline & OR $(95 \% \mathrm{CI})$ & $P$ Value & OR $(95 \% \mathrm{CI})$ & $P$ Value \\
\hline Age, younger than $80 \mathrm{yr}$ & $0.71(0.42-1.20)$ & .200 & $1.53(0.96-2.43)$ & .075 \\
\hline Sex, female & $0.94(0.59-1.48)$ & .772 & $1.46(0.96-2.23)$ & .078 \\
\hline Race, black & $2.34(1.31-4.17)$ & .004 & $1.16(0.66-2.05)$ & .615 \\
\hline $\mathrm{MHI}<\$ 80,000$ & $0.71(0.45-1.14)$ & .160 & $1.25(0.82-1.90)$ & .296 \\
\hline $\mathrm{NIHSS} \geq 10$ & $1.72(1.01-2.92)$ & .045 & $0.10(0.06-0.17)$ & $<.001$ \\
\hline Baseline ambulatory & $4.34(1.77-10.66)$ & .001 & $1.41(0.69-2.86)$ & .349 \\
\hline Onset-to-arrival $>6 \mathrm{hr}$ & $1.98(1.14-3.43)$ & .015 & $0.64(0.39-1.03)$ & .063 \\
\hline $2019-2020$ & $0.34(0.21-0.55)$ & $<.001$ & $0.73(0.47-1.11)$ & .141 \\
\hline Off-hours & $1.14(0.71-1.82)$ & .588 & $0.80(0.52-1.23)$ & .294 \\
\hline Arrival by ambulance & $1.15(0.66-1.98)$ & .628 & $0.49(0.32-0.77)$ & .002 \\
\hline PSC-G & $9.40(5.76-15.34)$ & $<.001$ & $0.85(0.49-1.49)$ & .571 \\
\hline IV rtPA & $2.27(1.33-3.87)$ & .003 & $1.80(1.07-3.03)$ & .026 \\
\hline Thrombectomy & $0.68(0.37-1.25)$ & .215 & $0.54(0.24-1.20)$ & .129 \\
\hline Rescan delay & NA & NA & $0.53(0.30-0.95)$ & .032 \\
\hline
\end{tabular}

Note:-MHI indicates median household income, NA; not applicable.

patients for both the CSC-G and PSC-G. The univariate and regression analyses were performed separately for these alternate scenarios (1- and 6-hour time limits). Furthermore, distinct bivariate and regression analyses were conducted for the CSC-G and PSC-G separately. Statistical analyses were performed using SAS, Version 9.4 (SAS Institute). A $P$ value $<.05$ was considered statistically significant.

\section{RESULTS}

During the study period, a total of 2216 consecutive patients with ischemic stroke were admitted to the comprehensive stroke center. Of these, $26.5 \%$ (588/2216 patients) met the inclusion criteria, comprising $66.3 \%$ (390/588) of patients in the CSC-G and $33.7 \%(198 / 588)$ of patients in the PSC-G (Fig 1). The mean age of the study cohort was 71.7 (SD, 15.0) years with range of $22-101$ years. The cohort comprised $46.4 \%$ women, $57.7 \%$ white, $17.0 \%$ black, and $12.4 \%$ Asian patients. The Online Supplemental Data provide the demographic and clinical characteristics of the study population.

The cohort was stratified into patients with and without rescan time delays ( $\geq 10$ minutes), of which $27.9 \%$ (164/588) were classified with a rescan time delay with a mean of 53.7 (SD, 43.4) minutes and range of 10-224 minutes. Comparison of patients with and without rescan time delays (Online Supplemental Data) revealed statistically significant differences in race, arrival year, arrival time and mode, imaging location, baseline function, and stroke severity. Specifically, the rescan time delay group had a significantly higher proportion of black patients, arrival at off-hours, arrival by ambulance, PSC-G imaging location, prestroke independent ambulation ( $\mathrm{mRS} \leq 3$ ), and severe stroke presentation (NIHSS $\geq 10$ ). Insurance status and median household income were not significantly different among groups. Acute stroke treatment, particularly with intravenous thrombolysis at the comprehensive stroke center and thrombectomy, was significantly more frequent in the rescan time delay group. Patients with rescan time delays were significantly more likely to have longer hospital admissions (length of hospital stay, $\geq 8$ days), while discharge home was significantly more likely in those without rescan time delays $(46.5 \%$ versus $26.8 \%, P<.001$ ). Finally, the frequency of rescan delays significantly decreased in 2019-2020 compared with 2017-2018 (16.9\% [49/290 patients] versus 38.6\% [115/298 patients], $P<.001)$.

The mean overall time difference calculated between NCCT and CTA \pm CTP was significantly higher in PSC-G compared with CSC-G $(n=588 ; 40.4$ [SD, 46.4] minutes versus 3.9 [SD, 9.0] minutes, $P<.001)$. Similarly, in the subset of patients with rescan time delays $(n=164)$, the mean delay duration was significantly higher in PSC-G compared with CSC-G (65.4 [SD, 45.4] minutes versus 23.6 [SD, 14.0] minutes, $P<.001)$. Secondary analyses of the alternate scenarios applying uniform inclusion criteria of 1and 6-hour time limits for both CSC-G and PSC-G showed findings consistent with the primary analysis (Online Supplemental Data). Figure 2 plots the distribution of overall time differences between NCCT and CTA \pm CTP, comparing PSC-G and CSC-G in the primary and secondary inclusion scenarios.

Several factors were found to be independently associated with rescan time delay in the multivariable logistic regression analysis (Table). Acute stroke imaging at a primary stroke center was the most significant factor associated with rescan time delay (OR $=9.40$; 95\% CI, 5.76-15.34; $P<.001)$. Other factors associated with increased odds of rescan time delay included intravenous thrombolytic administration $(\mathrm{OR}=2.27$; 95\% CI, $1.33-$ $3.87 ; P=.003)$, black race $(\mathrm{OR}=2.34 ; 95 \% \mathrm{CI}, 1.31-4.17$; $P=.004)$, baseline independent ambulation $(\mathrm{OR}=4.34 ; 95 \% \mathrm{CI}$, $1.77-10.66 ; P=.001)$, admission NIHSS $\geq 10$ (OR $=1.72 ; 95 \%$ CI, 1.01-2.92; $P=.045$ ), and onset-to-arrival time of $>6$ hours $(\mathrm{OR}=1.98 ; 95 \% \mathrm{CI}, 1.14-3.43 ; P=.015)$. However, admission in 2019-2020 was associated with significantly decreased odds of rescan time delay $(\mathrm{OR}=0.34 ; 95 \% \mathrm{CI}, 0.21-0.55 ; P<.001)$.

When we controlled for factors associated with outcome, such as advanced age, baseline NIHSS, and treatment, additional analyses revealed that rescan time delays were significantly associated with decreased odds of home discharge (OR $=0.53$; $95 \% \mathrm{CI}$, 0.30-0.95; $P=.032$ ). Furthermore, the odds of home discharge were significantly increased by association with intravenous thrombolysis and were decreased by association with an admission NIHSS score of $\geq 10$ and hospital arrival by ambulance. More details on this logistic regression analysis may be seen in 

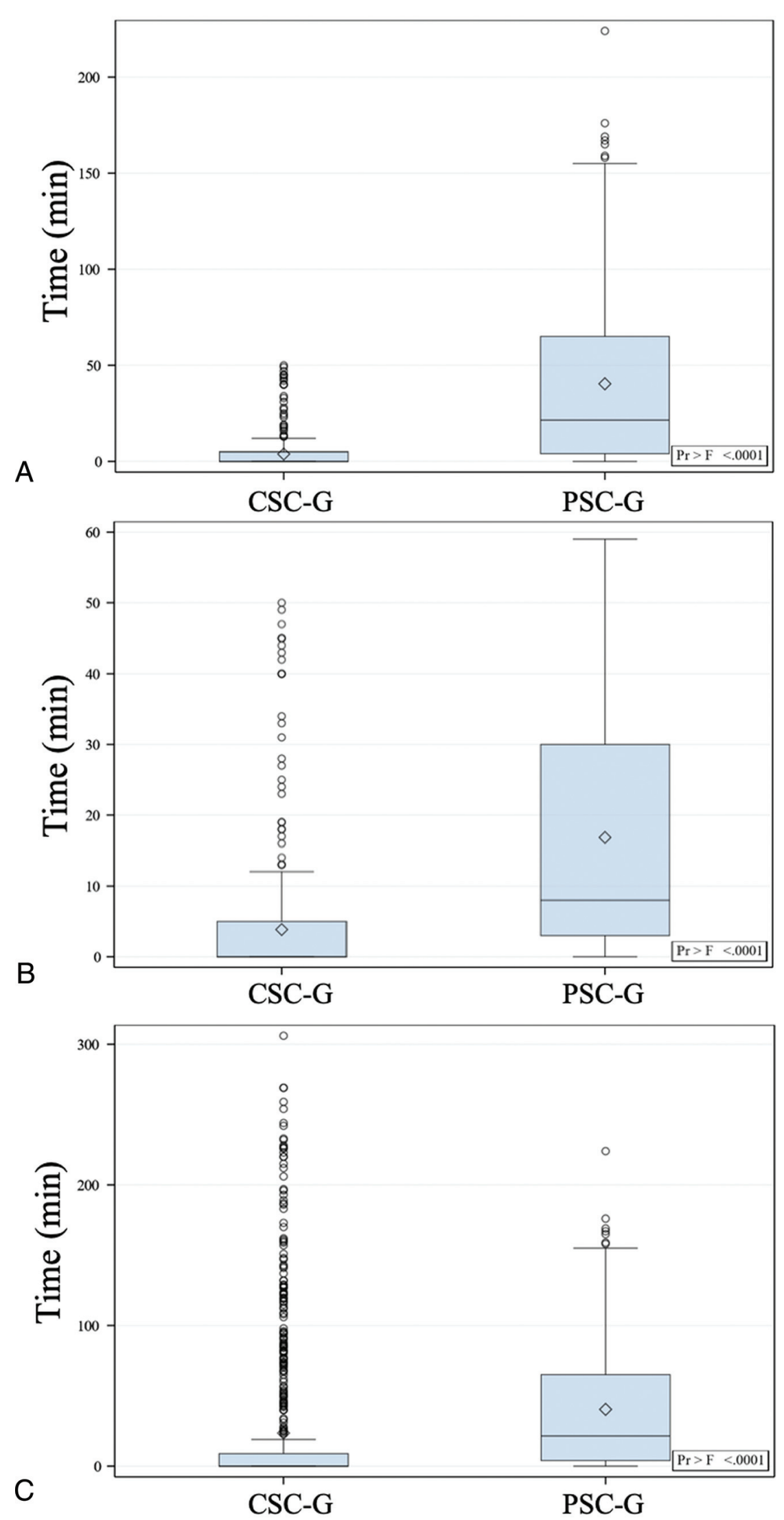

FIG 2. Distribution of overall time differences between NCCT and CTA \pm CTP scans stratified by CSC-G and PSC-G. A, Primary cohort. B, One-hour imaging window scenario. C, Six-hour imaging window scenario. the Table. Figure 3 shows the shift of discharge mRS scores and disposition by rescan time-delay intervals. Rescan time delays of $<20$ minutes were significantly associated with an increased proportion of patients who were functionally independent (mRS 0-2; $P=.030)$ at discharge. Furthermore, no rescan time delay was significantly associated with more patients discharged home $(P=.002)$.

After analyzing the alternate inclusion scenarios, we found similar findings with only a few differences for the rescan time delay and early outcome regression models compared with the primary analyses. These differences are presented in the Online Supplemental Data.

\section{DISCUSSION}

To our knowledge, this is the first study to quantify time delays associated with rescanning for advanced CT-based imaging following an initial NCCT in patients with acute ischemic stroke undergoing evaluation for acute treatment. Because a sequential approach to early CT-based imaging is recommended in current guidelines for patients being considered for thrombectomy, understanding the time delays associated with this approach is important because successful reperfusion and good clinical outcomes are timedependent. While almost three-quarters of our acute ischemic stroke cohort underwent NCCT and CTA \pm CTP simultaneously, not doing so was associated with an average of 53.7 (SD, 43.4) minutes of time delay between imaging. Rescanning was significantly more common and the mean delay 2.8 times longer at primary stroke centers compared with the comprehensive stroke center, a finding that remained significant after controlling for other demographic and clinical factors. Even the lower mean time-delay duration observed in CSC-G (23.6 minutes) accounts for $26 \%$ of the 90 -minute door-to-device goal for direct arrivals in the Target: Stroke Phase III initiative.

Nonetheless, the frequency of rescanning significantly decreased during the study period, owing to the 


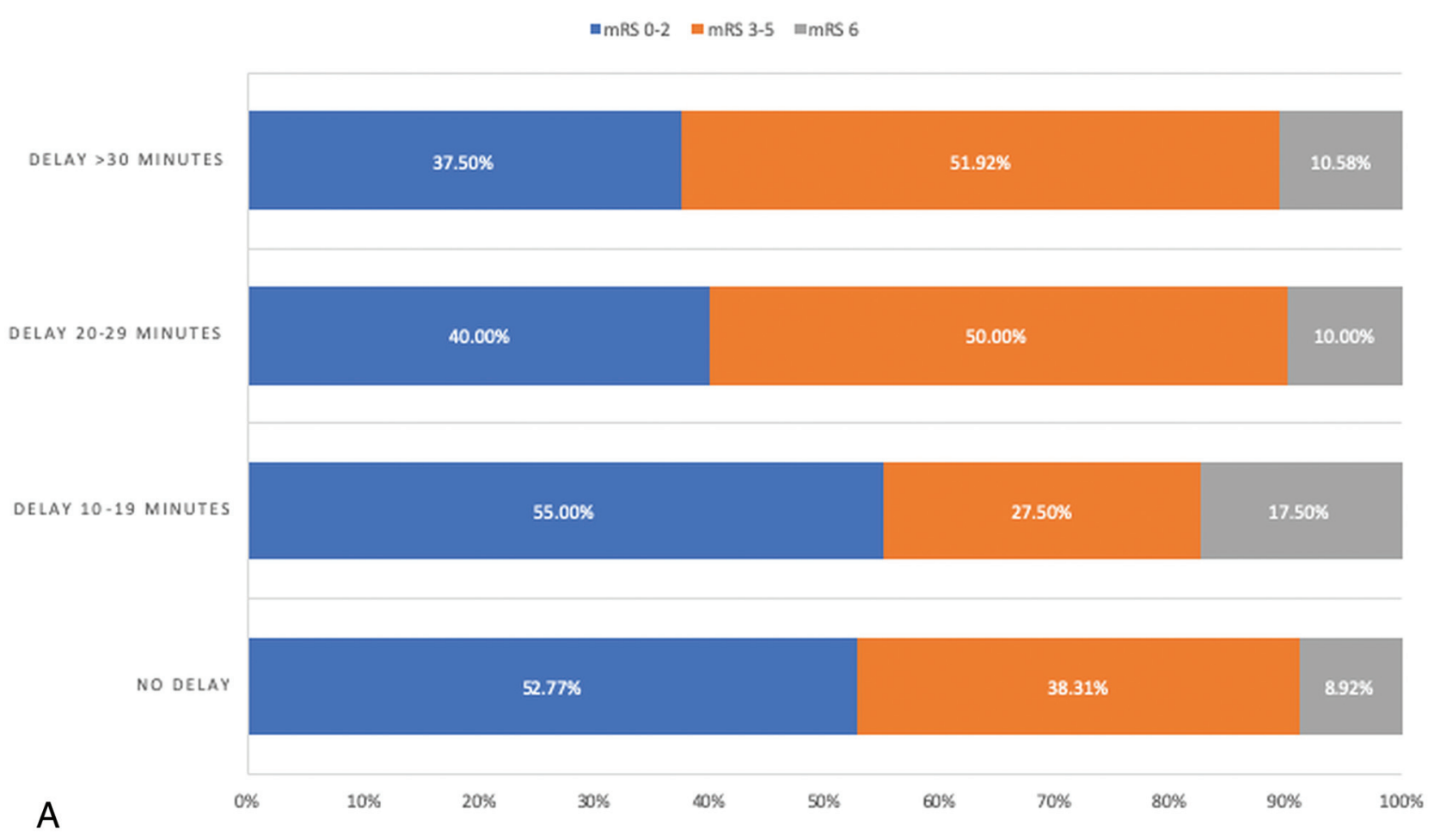

\section{DISCHARGE DISPOSITION}

घHome $n$ Rehabilitation $=$ Expired/Hospice

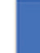

$$
25.00 \%
$$

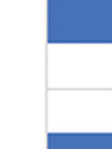

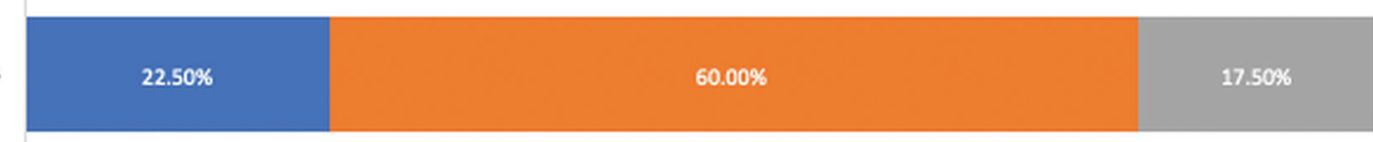

NO DELAY

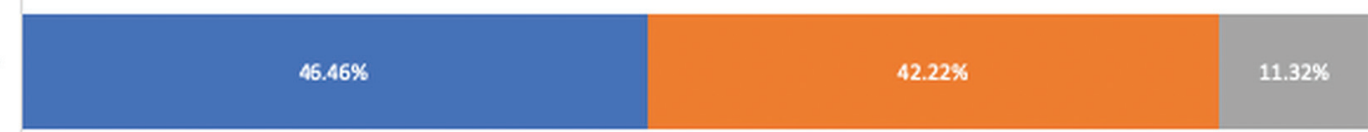

B

$0 \%$

$10 \%$

$20 \%$

$30 \%$

$40 \%$

$50 \%$

$60 \%$

$80 \%$

$90 \%$

$100 \%$

FIG 3. Distribution of $\mathrm{mRS}$ score $(A)$ and discharge disposition (B) by time-delay intervals.

adoption of a new workflow at the comprehensive stroke center for early CTAP in all patients with stroke presenting in the 24hour treatment window and protocolized early CTA use at many primary stroke centers. To improve efficiencies even further, some have recently advocated a direct-to-angiography suite workflow, bypassing the CT scanner in an emergency department entirely, 
with CTA \pm CTP performed in the interventional suite using conebeam CT technology. ${ }^{31-33}$ Two observational studies ${ }^{31,32}$ have shown improved time to treatment with this novel paradigm; however, improved functional outcomes, or even workflow efficiencies, were not proved in a randomized trial. ${ }^{34}$

As might be expected, patients administered intravenous thrombolytics or presenting $>6$ hours from last known well were significantly more likely to have time delays to CTA \pm CTP. In the multivariable logistic regression analysis, endovascular treatment was not associated with time delays after controlling for the other factors listed in the Table. The observed independent association between intravenous thrombolytic administration and time delay was likely a result of workflow when thrombolytics were administered immediately post-NCCT, but before CTA \pm CTP, especially when treatment was delivered outside the CT suite. Less-expected findings included racial disparity of increased time delays in black patients, particularly in the PSC-G, and increased time delays in patients with higher admission NIHSS scores and those ambulatory at baseline. The time delays in black patients were consistent across both secondary analyses using identical inclusion windows for the CSC-G and PSC-G and even when controlling for median household income. Delays in stroke care experienced by black patients in emergency departments have been reported by others. ${ }^{35}$ Bias in care delivery, as well as delayed stroke-symptom recognition and hospital arrival, and less use of ambulance services by black patients may be contributory. ${ }^{28}$ The relation to higher admission NIHSS scores may result from causes of delay that we could explain, such as blood pressure instability, airway management, or treatment for agitation that may occur in patients with more severe deficits.

Delay between NCCT and CTA \pm CTP was associated with a near $50 \%$ reduction in the likelihood of home discharge, and scan time differences of $<20$ minutes were associated with a higher proportion of patients who were functionally independent at discharge. The magnitude of time delay found in our study would be expected to significantly impact outcome following successful reperfusion. For instance, based on data from the Interventional Management of Stroke (IMS) III Trial, the mean delay we found translated to a $21 \%$ reduction in the relative likelihood of 90 -day functional independence. ${ }^{10}$ Furthermore, according to data from SWIFT-STAR trials ${ }^{9}$ and HERMES meta-analysis, ${ }^{8}$ our finding would yield $12 \%-14 \%$ additional patients having more disabled outcomes post-successful reperfusion and $9 \%$ fewer patients achieving 90-day functional independence. ${ }^{8}$ Similarly, per the Multicenter Randomized Clinical Trial of Endovascular Treatment of Acute Ischemic Stroke in the Netherlands (MR CLEAN) registry, delay associated with rescanning would reduce the probability of 90 -day functional independence by $7 \% .^{11}$

Our study has several strengths and limitations. We aimed to focus our inclusion criteria on patients who were most likely undergoing evaluation for endovascular treatment and not purely for secondary stroke-prevention evaluation; this feature is a strength of this study because delayed imaging is not a main concern to patients outside endovascular treatment considerations. Nonetheless, this goal necessitated different inclusion time windows for patient selection in the PSC-G and CSC-G, which could have introduced selection bias into our results. We attempted to mitigate this limitation by re-analyzing our data using uniform criteria applied to both the PSC-G and CSC-G. These alternate inclusion scenarios (1- and 6-hour time limits) showed very similar patterns of results compared with the primary analysis, though the magnitude of the time delay was certainly influenced by our inclusion time limits. Another strength is our inclusion and analysis of data from both primary and comprehensive stroke centers. This feature enabled us to compare time delays at different sites with varied resources and levels of stroke care. However, because our findings are from a single stroke network, broad applicability is limited and similar studies from other health care networks and geographic areas may be useful.

Our study has other important limitations. First, it has a retrospective study design; thus, we used discharge disposition and discharge mRS, instead of 90-day mRS as outcome measures because they were readily available in our database. Several studies support discharge disposition being a good predictor of a longer-term stroke outcome. ${ }^{36,37}$ Our retrospective design also limited our ability to include imaging results in our analyses, such as the presence of a large-vessel occlusion or a perfusion defect. Second, our sample size of 588 patients is relatively small and may have resulted in not detecting statistically small differences between groups. This sample size also precluded us from drawing conclusions from important subgroup analyses, such as those who underwent endovascular thrombectomy or had largevessel occlusion, and from stratifying by infarction vascular territory, because, for instance, patients with posterior circulation stroke may have more unstable conditions and may need airway management, resulting in increased time delays. Nonetheless, the ratio of the number of participants to the number of variables in our regression model is comparable with that in other studies.

\section{CONCLUSIONS}

Obtaining NCCT and CTA \pm CTP separately was associated with significant time delays in acquiring comprehensive CT imaging information for treatment decision-making in patients with acute ischemic stroke. Time delays to CTA \pm CTP were associated with poorer early clinical outcomes. Both intravenous thrombolytic administration and delayed onset-to-arrival times were independently associated with rescanning imaging time delays, while implementing protocols to obtain NCCT and CTAP simultaneously was associated with avoiding the observed time delays associated with rescanning. Considering that this imaging paradigm has been shown to be the most cost-effective imaging strategy for patients with acute ischemic stroke, ${ }^{38}$ the current study provides further support for simultaneous NCCT and CTA \pm CTP imaging to minimize time delays to treatment.

Disclosures: Jeffrey M. Katz-RELATED: Grant: National Institutes of Health/National Institute of Neurological Disorders and Stroke and Siemens*; UNRELATED: Expert Testimony: multiple law firms and malpractice insurance companies; Grants/Grants Pending: Medtronic*; Travel/Accommodations/Meeting Expenses Unrelated to Activities Listed: Medtronic. Artem T. Boltyenkov—RELATED: Grant: National Institute of Neurological Disorders and Stroke of the National Institutes of Health, Comments: award number R56NS114275*; UNRELATED: Employment: Siemens, Comments: Artem T. Boltyenkov is a full-time employee of Siemens; Stock/Stock Options: Siemens, Comments: Artem T. Boltyenkov is a shareholder of Siemens; OTHER RELATIONSHIPS: Artem T. Boltyenkov is a visiting scholar at Northwell Health. Gabriela Martinez-RELATED: Grant: National Institutes of Health*; 
UNRELATED: Employment: Siemens; Stock/Stock Options: Siemens. Joseph O'HaraRELATED: Grant: National Institutes of Health, Siemens, Comments: Research reported in this publication was supported by the Feinstein Institutes for Medical Research, Northwell Health, the National Institute of Neurological Disorders and Stroke of the National Institutes of Health under award number R56NS114275, and Siemens.* Chinara Feizullayeva_RELATED: Grant: National Institutes of Health and Siemens. Ankur Pandya-RELATED: Grant: Siemens (Feinstein Institutes for Medical Research). Pina C. Sanelli-RELATED: Grant. National Institute of Neurological Disease and Stroke, Siemens. * Money paid to the institution.

\section{REFERENCES}

1. Goyal M, Demchuk AM, Menon BK, et al. Randomized assessment of rapid endovascular treatment of ischemic stroke. $N$ Engl J Med 2015;372:1019-30 CrossRef Medline

2. Campbell BC, Mitchell PJ, Kleinig TJ, et al. Endovascular therapy for ischemic stroke with perfusion-imaging selection. $N$ Engl J Med 2015;372:1009-18 CrossRef Medline

3. Berkhemer OA, Fransen PSS, Beumer LA, et al. MR CLEAN Investigators. A randomized trial of intraarterial treatment for acute ischemic stroke. $N$ Engl J Med 2015;372:11-20 CrossRef Medline

4. Jovin T, Chamorro A, Cobo E, et al. REVASCAT Trial Investigators. Thrombectomy within 8 hours after symptom onset in ischemic stroke. N Engl J Med 2015;372:2296-2306 CrossRef Medline

5. Saver JL, Goyal M, Bonafe A, et al. Stent-retriever thrombectomy after intravenous t-PA vs. t-PA alone in stroke. $N$ Engl $\mathrm{J} \mathrm{Med}$ 2015;372:2285-95 CrossRef Medline

6. Nogueira RG, Jadhav AP, Haussen DC, et al. Thrombectomy 6 to 24 hours after stroke with a mismatch between deficit and infarct. $N$ Engl J Med 2018;378:11-21 CrossRef Medline

7. Albers GW, Marks MP, Kemp S, et al. Thrombectomy for stroke at 6 to 16 hours with selection by perfusion imaging. $N$ Engl J Med 2018;378:708-18 CrossRef Medline

8. Saver JL, Goyal M, van der Lugt A, et al. HERMES Collaborators. Time to treatment with endovascular thrombectomy and outcomes from ischemic stroke: a meta-analysis. JAMA 2016;316:1279-88 CrossRef Medline

9. Sheth SA, Jahan R, Gralla J, et al. for the SWIFT-STAR Trialists. Time to endovascular reperfusion and degree of disability in acute stroke. Ann Neurol 2015;78:584-93 CrossRef Medline

10. Khatri P, Yeatts SD, Mazighi M, et al. IMS III Trialists. Time to angiographic reperfusion and clinical outcome after acute ischaemic stroke: an analysis of data from the Interventional Management of Stroke (IMS III) phase 3 trial. Lancet Neurol 2014;13:567-74 CrossRef Medline

11. Mulder MJH, Jansen IG, Goldhoorn RB, et al. MR CLEAN Registry Investigators. Time to endovascular treatment and outcome in acute ischemic stroke: MR CLEAN registry results. Circulation 2018;138:232-40 CrossRef Medline

12. American Heart Association. Target: Stroke Phase III. https://www. heart.org/en/professional/quality-improvement/target-stroke/ introducing-target-stroke-phase-iii. Accessed January 5, 2021

13. Powers WJ, Rabinstein AA, Ackerson T, et al. Guidelines for the Early Management of Patients With Acute Ischemic Stroke: 2019 Update to the 2018 Guidelines for the Early Management of Acute Ischemic Stroke-A Guideline for Healthcare Professionals from the American Heart Association/American Stroke Association. Stroke 2019;50:e344-418 CrossRef Medline

14. Pierot L, Jayaraman MV, Szikora I, et al. Asian-Australian Federation of Interventional and Therapeutic Neuroradiology (AAFITN), Australian and New Zealand Society of Neuroradiology (ANZSNR), American Society of Neuroradiology (ASNR), Canadian Society of Neuroradiology (CSNR), European Society of Minimally Invasive Neurological Therapy (ESMINT), European Society of Neuroradiology (ESNR), European Stroke Organization (ESO), Japanese Society for NeuroEndovascular Therapy (JSNET), The French Society of Neuroradiology (SFNR), Ibero-Latin American Society of Diagnostic and Therapeutic Neuroradiology (SILAN), Society of NeuroInterventional Surgery (SNIS), Society of Vascular and Interventional Neurology (SVIN), World Stroke Organization (WSO), World Federation of Interventional Neuroradiology (WFITN). Standards of practice in acute ischemic stroke intervention: international recommendations. I Neurointerv Surg 2018;10:1121-26 CrossRef Medline

15. Wintermark M, Sanelli PC, Albers GW, et al. Society of NeuroInterventional Surgery. Imaging recommendations for acute stroke and transient ischemic attack patients: a joint statement by the American Society of Neuroradiology, the American College of Radiology and the Society of NeuroInterventional Surgery. J Am Coll Radiology 2013;10:828-32 CrossRef Medline

16. Kwei KT, Liang J, Wilson N, et al. Stroke severity affects timing time from stroke code activation to initial imaging is longer in patients with milder strokes. Neurologist 2018;23:79-82 CrossRef Medline

17. Mowla A, Doyle J, Lail NS, et al. Delays in door-to-needle time for acute ischemic stroke in the emergency department: a comprehensive stroke center experience. J Neurol Sci 2017;376:102-05 CrossRef Medline

18. Prabhakaran S, Khorzad R, Brown A, et al. Academic-community hospital comparison of vulnerabilities in door-to-needle process for acute ischemic stroke. Circ Cardiovasc Qual Outcomes 2015;8: S148-54 CrossRef Medline

19. Koge J, Matsumoto S, Nakahara I, et al. Improving treatment times for patients with in-hospital stroke using a standardized protocol. J Neurol Sci 2017;381:68-73 CrossRef Medline

20. Campbell BC, Christensen S, Levi CR, et al. Comparison of computed tomography perfusion and magnetic resonance imaging perfusiondiffusion mismatch in ischemic stroke. Stroke 2012;43:2648-53 CrossRef Medline

21. Eastwood JD, Lev MH, Wintermark M, et al. Correlation of early dynamic CT perfusion imaging with whole-brain MR diffusion and perfusion imaging in acute hemispheric stroke. AJNR Am J Neuroradiol 2003;24:1869-75 Medline

22. Saur D, Kucinski T, Grzyska U, et al. Sensitivity and interrater agreement of CT and diffusion-weighted MR imaging in hyperacute stroke. AJNR Am J Neuroradiol 2003;24:878-85 Medline

23. Urbach $H$, Flacke $S$, Keller E, et al. Detectability and detection rate of acute cerebral hemisphere infarcts on CT and diffusionweighted MRI. Neuroradiol 2000;42:722-27 CrossRef Medline

24. United States Census Bureau. B19013 median household income in the past 12 months (in 2019 inflation-adjusted dollars). https:// censusreporter.org/tables/B19013/. Accessed September 15, 2020

25. Saposnik G, Cote R, Phillips S, et al. Stroke outcome in those over 80: a multicenter cohort study across Canada. Stroke 2008;39:2310 17 CrossRef Medline

26. Arora R, Salamon E, Katz JM, et al. Use and outcomes of intravenous thrombolysis for acute ischemic stroke in patients $\mathbf{2 9 0}$ years of age. Stroke 2016;47:2347-54 CrossRef Medline

27. NIH policy on reporting race and ethnicity data: subjects in clinical research. Release Date. August 8, 2001. https://grants.nih. gov/grants/guide/notice-files/not-od-01-053.html. Accessed April 22, 2021

28. Cruz-Flores S, Rabinstein A, Biller J, et al. Council on Quality of Care and Outcomes Research. Racial-ethnic disparities in stroke care: the American experience-a statement for healthcare professionals from the American Heart Association/American Stroke Association. Stroke 2011;42:2091-116 CrossRef Medline

29. Goyal M, Almekhlafi MA, Fan L, et al. Evaluation of interval times from onset to reperfusion in patients undergoing endovascular therapy in the IMS III Trial. Circulation 2014;130:265-72 CrossRef Medline

30. Campbell BC, Weir L, Desmond PM, et al. CT perfusion improves diagnostic accuracy and confidence in acute ischaemic stroke. $J$ Neurol Neurosurg Psychiatry 2013;84:613-18 CrossRef Medline

31. Ribó M, Boned S, Rubiera M, et al. Direct transfer to angiosuite to reduce door-to-puncture time in thrombectomy for acute stroke. $J$ Neurointerv Surg 2018;10:221-24 CrossRef Medline 
32. Mendez B, Requena M, Aires A, et al. Direct transfer to angio-suite to reduce workflow times and increase favorable clinical outcome. Stroke 2018;49:2723-27 CrossRef Medline

33. Brehm A, Tsogkas I, Maier IL, et al. One-stop management with perfusion for transfer patients with stroke due to a large-vessel occlusion: feasibility and effects on in-hospital times. AJNR Am J Neuroradiol 2019;40:1330-34 CrossRef Medline

34. Pfaff JA, Schönenberger S, Herweh C, et al. Direct transfer to angiosuite versus computed tomography-transit in patients receiving mechanical thrombectomy: a randomized trial. Stroke 2020;51:2630-38 CrossRef Medline

35. Karve SJ, Balkrishnan R, Mohammad YM, et al. Racial/ethnic disparities in emergency department waiting time for stroke patients in the United States. J Stroke Cerebrovasc Dis 2011;20:30-40 CrossRef Medline

36. Belagaje SR, Sun CH, Nogueira RG, et al. Discharge disposition to skilled nursing facility after endovascular reperfusion therapy predicts a poor prognosis. J Neurointerv Surg 2015;7:99-103 CrossRef Medline

37. Bautista AE, Meyer DM, Meyer BC. Novel definition of stroke “good responders" predicts 90-day outcome after thrombolysis. J Stroke Cerebrovasc Dis 2019;28:104422 CrossRef Medline

38. Martinez G, Katz JM, Pandya A, et al. Cost-effectiveness study of initial imaging selection in acute ischemic stroke care. J Am Coll Radiol 202031362-64 CrossRef Medline 